PROCEEDINGS OF THE

AMERICAN MATHEMATICAL SOCIETY

Volume 132, Number 6, Pages 1589-1597

S 0002-9939(03)07282-4

Article electronically published on October 21, 2003

\title{
A LINEAR FUNCTION ASSOCIATED TO ASYMPTOTIC PRIME DIVISORS
}

\author{
DANIEL KATZ AND ERIC WEST
}

(Communicated by Bernd Ulrich)

\begin{abstract}
Let $R$ be a Noetherian standard $\mathbb{N}^{d}$-graded ring and $M, N$ finitely generated, $\mathbb{N}^{d}$-graded $R$-modules. Let $I_{1}, \ldots, I_{s}$ be finitely many homogeneous ideals of $R$. We show that there exist linear functions $f, g: \mathbb{N}^{s} \rightarrow$ $\mathbb{N}^{d}$ such that the associated primes over $R_{0}$ of $\left[\operatorname{Ext}^{i}\left(N, M / I_{1}^{n_{1}} \cdots I_{s}^{n_{s}} M\right)\right]_{m}$ and $\left[\operatorname{Tor}_{i}\left(N, M / I_{1}^{n_{1}} \cdots I_{s}^{n_{s}} M\right)\right]_{m}$ are stable whenever $m \in \mathbb{N}^{d}$ satisfies $m \geq$ $f\left(n_{1}, \ldots, n_{s}\right)$ and $m \geq g\left(n_{1}, \ldots, n_{s}\right)$, respectively.
\end{abstract}

\section{INTRODUCTION}

In this paper we bring together a couple of themes in the asymptotic theory of ideals. The first of these themes concerns the study of asymptotic prime divisors, i.e., the study of prime ideals associated to a family of ideals or modules indexed by increasing parameters. In [B], Brodmann showed that for a Noetherian ring $R$, an ideal $I \subseteq R$ and a finitely generated $R$-module $M$, the associated primes $\operatorname{Ass}_{R}\left(M / I^{n} M\right)$ stabilize for $n$ sufficiently large. This result can be derived from a stability result for graded modules: If $R$ is a standard graded ring and $M$ a finitely generated graded $R$-module, then $\operatorname{Ass}_{R_{0}}\left(M_{n}\right)$ is stable for $n$ large (see $[\mathrm{Mc})$. Brodmann's result was extended to finitely many ideals containing regular elements (and $M=R$ ) in KMR. Recently, Sharp and Kingsbury extended this last result to modules and an arbitrary finite collection of ideals (see $[\mathrm{KS}]$ ). Inspired by this, in [W] the second author initiated a multigraded theory that extends the graded results in $\mathrm{Mc}$ and recovers cleanly the results from $\mathrm{KMR}$ and $\overline{\mathrm{KS}}$.

The second theme in this paper is that of showing the existence of a linear function derived from properties of powers of ideals. One of the first (and most beautiful) of recent results along these lines is the main result of $\mathrm{Sw}$. There, it is shown that if $R$ is a Noetherian ring and $I \subseteq R$ is an ideal, then there exist $k$ independent of $n$ and a primary decomposition of $I^{n}$ such that the radicals of the components in the decomposition raised to the $k n$th power belong to the corresponding components. This too was extended to many ideals in [Sh]. The linear functions in $[\mathrm{Sw}$ and $[\mathrm{Sh}$ are derived from properties of powers of the ideal. In $[\mathrm{Ko}$ and $[\mathrm{CHT}]$ it is shown that, in the graded case, the Castelnuovo regularity of $I^{n}$ is eventually given by a linear function. This is an example of a linear function dependent on the powers of a homogeneous ideal, but derived from properties of the

Received by the editors April 8, 2002 and, in revised form, February 13, 2003.

2000 Mathematics Subject Classification. Primary 13A02, 13A15, 13A30, 13E05.

Key words and phrases. Associated prime, multi-graded module, homology module. 
grading. The linear functions we introduce in this paper are also power dependent and derived from the grading.

Suppose that $R$ is a graded or multigraded ring, $I_{1}, \ldots, I_{s}$ homogeneous ideals, and $M$ a finitely generated (appropriately) graded $R$-module. For each $n_{1}, \ldots, n_{s}$, $M / I^{n_{1}} \cdots I^{n_{s}} M$ is a graded or multigraded $R$-module. Thus we can consider the graded pieces of these modules over the zero part of the ring. It follows from the main result in this paper that there exists a linear function $h$ of $n_{1}, \ldots, n_{s}$ so that the $R_{0}$ primes associated to the graded components of $M / I^{n_{1}} \cdots I^{n_{s}} M$ stabilize for values of the grading larger than $h\left(n_{1}, \ldots, n_{s}\right)$. In fact, it is not much harder to prove this result for certain homology modules derived from $I_{1}^{n_{1}} \cdots I_{s}^{n_{s}}$; so we do this instead. Our main result is facilitated by two new observations of independent interest. The first is that the associated primes of the homology modules derived from $I_{1}^{n_{1}} \cdots I_{s}^{n_{s}}$ stabilize for $n_{i}$ sufficiently large. The second observation is the following: In the graded case, if $A$ is a finitely generated graded (or multigraded) module over $R$, then an explicit value of $m$ can be given beyond which the primes in $A s s_{R_{0}}\left(A_{m}\right)$ are stable.

\section{Notation}

Throughout this paper $R$ will denote a Noetherian, commutative ring with identity. Unless stated otherwise, all $R$-modules will be finitely generated. We will deal with rings and modules graded by $\mathbb{N}^{d}$, where, by abuse of notation, $\mathbb{N}$ denotes the set of nonnegative integers, and $d$ is any fixed positive integer. For convenience, we may sometimes omit the reference to $\mathbb{N}^{d}$ in our notation. In particular, if $R$ is an $\mathbb{N}^{d}$-graded ring, then it should be assumed that any graded $R$-module or homogeneous ideal is graded by $\mathbb{N}^{d}$ as well. We use the term "standard" in the sense of Stanley, i.e., a standard $\mathbb{N}^{d}$-graded ring is one that is generated in total degree one.

We will be working with $d$-tuples from $\mathbb{N}^{d}$ and $s$-tuples from $\mathbb{N}^{s}$, the latter occurring almost always as superscripts in a power product of $s$ ideals. Rather than burden the reader with excessive notation, we will simplify our notation and use $m \in \mathbb{N}^{d}$ or $n \in \mathbb{N}^{s}$ to indicate $d$-tuples or $s$-tuples. We will also abbreviate a power product of ideals $I_{1}^{n_{1}} \cdots I_{s}^{n_{s}}$ in $R$ by $I^{n}$, for $n=\left(n_{1}, \ldots, n_{s}\right) \in \mathbb{N}^{s}$. Thus, for example, if $R$ is an $\mathbb{N}^{d}$-graded ring, $I_{1}, \ldots, I_{s} \subseteq R$ homogeneous ideals, and $M$ a graded $R$-module, then for $m \in \mathbb{N}^{d}$ and $n \in \mathbb{N}^{s}$ we will write $\left[I^{n} M\right]_{m}$ for the degree $m$ component of the $\mathbb{N}^{d}$-graded module $I^{n_{1}} \cdots I^{n_{s}} M$. We will use subscripts to denote components of our $d$-tuples or $s$-tuples. Thus $m_{i}$ means the $i$ th component of $m=\left(m_{1}, \ldots, m_{d}\right) \in \mathbb{N}^{d}$. Superscripts will be used to indicate lists of $d$-tuples or $s$-tuples. In particular, we will let $\left\{e^{j}\right\}$ denote the standard basis elements in $\mathbb{N}^{d}$ or $\mathbb{N}^{s}$.

We will often use the partial order on $\mathbb{N}^{d}$ defined by $n \geq m$ if and only if $n_{i} \geq m_{i}$ for all $1 \leq i \leq d$. We will denote by $\sup \left\{n^{1}, \ldots, n^{t}\right\}$ that $d$-tuple whose $i$ th coordinate is $\max \left\{\left(n^{1}\right)_{i}, \ldots,\left(n^{t}\right)_{i}\right\}$. Finally, for an $\mathbb{N}^{d}$-graded ring $R$, and an $\mathbb{N}^{d}$-graded $R$-module $M$, we will use the notation $A s s_{R}^{+}(M)$ to denote the set of relevant prime ideals in $A s s_{R}(M)$. That is, $P$ belongs to $A s s_{R}^{+}(M)$ if and only if $P \in \operatorname{Ass}_{R}(M)$ and $R_{e^{i}} \not \subset P$, for all $1 \leq i \leq d$. 


\section{Asymptotic stability of Associated primes OF HOMOLOGY MODULES}

In this section we will show that certain homology modules parameterized by $I_{1}^{n_{1}} \cdots I_{s}^{n_{s}}$ enjoy asymptotic stability of associated primes. $R$ will denote a (not necessarily graded) ring containing the ideals $I_{1}, \ldots, I_{s}$. As stated above, for $n$ in $\mathbb{N}^{s}$, we will write $I^{n}$ for $I_{1}^{n_{1}} \cdots I_{s}^{n_{s}}$. For $R$-modules $A^{\prime} \subseteq A, B^{\prime} \subseteq B, C^{\prime} \subseteq C$, we will consider homology modules of complexes of the form

$$
A / I^{n} A^{\prime} \rightarrow B / I^{n} B^{\prime} \rightarrow C / I^{n} C^{\prime} .
$$

For $n$ large, these homology modules can be written as $\left(U+I^{n-q} V\right) / I^{n-q} W$ where $U, V, W$ are submodules of a finitely generated $R$-module $T$ and $W \subseteq V$. Our task will be to show that $A s s_{R}\left(\left(U+I^{n} V\right) / I^{n} W\right)$ is stable for $n \in \mathbb{N}^{s}, n>>0$.

We start by reminding the reader of the following results in the theory of regular ideals.

Lemma 3.1. Let $R, T, W$ be as above. Suppose that $I_{1}, \ldots, I_{s}, J$ are ideals of $R$.

(1) If $I_{j}$ contains a $T$-regular element, then there exists $r_{j} \in \mathbb{N}$ such that for all $n \in \mathbb{N}^{s}$ satisfying $n_{j} \geq r_{j},\left(I^{n+e^{j}} W \quad:_{T} I_{j}\right)=I^{n} W$.

(2) If $J$ contains a T-regular element, then there exists $k \in \mathbb{N}^{s}$ such that for all $n \in \mathbb{N}^{s}$, satisfying $n \geq k,\left(I^{n} W:_{T} J\right)=I^{n-k}\left(I^{k} W:_{T} J\right)$.

Proof. For part (1), if $T=R$, this is Proposition 1.4 of $\mathrm{KMR}$. The extension to the module case is given by Lemma 1.3 of $\mathrm{KS}$. For the second part, if $x \in J$ is $T$-regular, then summing $\left(I^{n} W:_{T} x\right)$ over $n \in \mathbb{N}^{s}$ gives a finite module over the ring obtained by summing $I^{n}$ over $n$. Thus the submodule obtained by summing $\left(I^{n} W:_{T} J\right)$ is finite as well, and so the result follows by the $\mathbb{N}^{s}$-graded form of the Artin-Rees lemma.

Lemma 3.2. Let $R$ be a Noetherian ring, $I_{1}, \ldots, I_{s}$ ideals of $R$ and $U, V \subseteq T$ finitely generated $R$-modules. Fix a prime ideal $P$ of $R$ such that $P$ contains at least one $I_{j}$. Then there exists $n^{0} \in \mathbb{N}^{s}$ such that $\left(U+I^{n} V\right) \cap\left(0:_{T} P\right)=U \cap\left(0:_{T} P\right)$ for all $n \geq n^{0}$.

Proof. Write $Z:=\left(0::_{T} P\right)$. By the multigraded form of the Artin-Rees lemma, there exists $q \in \mathbb{N}^{s}$ such that $I^{n} V \cap(U+Z)=I^{n-q}\left(I^{q} V \cap(U+Z)\right)$, for all $n \geq q$. Set $n^{0}:=q+(1, \ldots, 1)$, take $n \geq n^{0}$ and let $u+v=z \in\left(U+I^{n} V\right) \cap Z$ with $u \in U$, $v \in I^{n} V$ and $z \in Z$. Then $-u+z \in(U+Z) \cap I^{n} V=I^{n-q}\left(I^{q} V \cap(U+Z)\right)$. Since $I_{j} \cdot Z=0$, it follows that $v=-u+z$ is contained in $U$. Thus, $u+v$ belongs to $U$, and therefore to $U \cap Z$, since we also have $z \in Z$.

For the next lemma, we note that for finite modules $U, V, W \subseteq T$, with $W \subseteq V$, the union over $n \in \mathbb{N}^{s}$ of the primes in $A s s\left(\left(U+I^{n} V\right) / I^{n} W\right)$ is finite since these are included among the primes in $\bigcup_{n \in \mathbb{N} s} A s s\left(T / I^{n} W\right)$, which is finite (see [KS]). We will also choose $q \in \mathbb{N}^{s}$ satisfying three conditions. That we can meet the first two conditions follows from Lemma 3.1 and the multigraded form of the Artin-Rees lemma.

Lemma 3.3. Let $R$ be a Noetherian ring, $I_{1}, \ldots, I_{s}$ ideals of $R$ and $W, V, U \subseteq$ $T$ finitely generated $R$-modules, with $W \subseteq V$. Let $q \in \mathbb{N}^{s}$ satisfy the following conditions: 
(1) For each $1 \leq j \leq s,\left(I^{n+e^{j}} W:_{T} I_{j}\right) \subseteq I^{n} W+N_{j}$ for all $n \in \mathbb{N}^{s}$ satisfying $n \geq q$, where $N_{j}$ denotes the set of elements of $T$ annihilated by some power of $I_{j}$.

(2) For all $1 \leq j \leq s, I^{n} T \cap N_{j}=0$, for all $n \in \mathbb{N}^{s}, n \geq q$.

(3) For any $n \geq q$ and $P \in A s s\left(\left(U+I^{n} V\right) / I^{n} W\right)$, if $I_{j} \subseteq P$ for some $1 \leq j \leq s$, then the conclusion of Lemma 3.2 holds.

For any $n \geq q$ and $P \in A s s\left(\left(U+I^{n} V\right) / I^{n} W\right)$, if $P=\left(I^{n} W: c\right)$, then for any $1 \leq j \leq s$, either $P=\left(I^{n+e^{j}} W: I_{j} c\right)$ or $P=\left(I^{n+e^{j}} W: a\right)=(0: a)$, with $a \in U \cap(0: T P)$.

Proof. We first note that if $I_{j} \not \subset P$, then clearly $P=\left(I^{n+e^{j}} W: I_{j} c\right)$. Suppose then that $I_{j} \subseteq P$ and $\left(I^{n+e^{j}} W: I_{j} c\right)$ strictly contains $P$. Take $r \in\left(I^{n+e^{j}} W: I_{j} c\right) \backslash P$. Thus, $r c \in\left(I^{n+e^{j}} W:_{T} I_{j}\right)$. By (1), we may write $r c=b+a$, with $b \in I^{n} W$ and $a \in N_{j}$. Thus, $P=\left(I^{n} W: c\right)=\left(I^{n} W: r c\right)=\left(I^{n} W: a\right)$. Because $a \in N_{j}$, we have by condition $(2)$ that $P=\left(I^{n} W: a\right)=(0: a)$. It follows readily from this that $P=\left(I^{n+e^{j}} W: a\right)$. Moreover, $a=r c-b \in\left(U+I^{n} V\right) \cap\left(0:_{T} P\right)$. Thus by (3), we have $a \in U \cap\left(0:_{T} P\right)$, which is what we want.

The following proposition is the main result of this section. Note that if we take $\alpha$ and $\beta$ in the proposition to be the zero maps, then we recover the results from KMR and $[\mathrm{KS}$ cited in the introduction.

Proposition 3.4. Let $R$ be a Noetherian ring, $I_{1}, \ldots, I_{s} \subseteq R$ ideals. Consider the complex $A \stackrel{\alpha}{\longrightarrow} B \stackrel{\beta}{\longrightarrow} C$ of finitely generated $R$-modules. Suppose $A^{\prime} \subseteq A, B^{\prime} \subseteq B$ and $C^{\prime} \subseteq C$ are submodules satisfying $\alpha\left(A^{\prime}\right) \subseteq B^{\prime}$ and $\beta\left(B^{\prime}\right) \subseteq C^{\prime}$. For $n \in \mathbb{N}^{s}$, let $H(n)$ denote the homology of the induced complex

$$
A / I^{n} A^{\prime} \stackrel{\alpha(n)}{\longrightarrow} B / I^{n} B^{\prime} \stackrel{\beta(n)}{\longrightarrow} C / I^{n} C^{\prime} .
$$

Then there exists $k \in \mathbb{N}^{s}$ such that $A s s_{R}(H(n))$ is stable for all $n \in \mathbb{N}^{s}, n \geq k$.

Proof. We first note that there exists $q \in \mathbb{N}^{s}$ and finitely generated $R$-modules $U, V, W \subseteq T$ with $W \subseteq V$ such that $H(n)$ is isomorphic to $\left(U+I^{n-q} V\right) / I^{n-q} W$ for all $n \in \mathbb{N}^{s}, n \geq q$. Indeed, extending the argument in [T, Proposition 3] to a finite collection of ideals, we take $q \in \mathbb{N}^{s}$ such that $I^{n} C^{\prime} \cap \operatorname{im}(\beta)=I^{n-q}\left(I^{q} C^{\prime} \cap \operatorname{im}(\beta)\right)$ holds for $n \geq q$. It follows that for such $n$,

$$
H(n)=\left(\operatorname{ker}(\beta)+I^{n-q} V^{\prime}\right) /\left(\operatorname{im}(\alpha)+I^{n-q} W^{\prime}\right),
$$

where $V^{\prime}=\beta^{-1}\left(I^{q} C^{\prime}\right)$ and $W^{\prime}=I^{q} B^{\prime}$. For $T:=B / \operatorname{im}(\alpha), U:=\operatorname{ker}(\beta) / \operatorname{im}(\alpha)$, $V:=\left(V^{\prime}+\operatorname{im}(\alpha)\right) / \operatorname{im}(\alpha)$ and $W:=\left(W^{\prime}+\operatorname{im}(\alpha)\right) / \operatorname{im}(\alpha), H(n)$ is isomorphic to $\left(U+I^{n-q} V\right) / I^{n-q} W$. Thus, it suffices to take any $R$-modules $U, V, W \subseteq T$, with $W \subseteq V$, and show that there exists $k \in \mathbb{N}^{s}$ such that $A s s_{R}\left(\left(U+I^{n} V\right) / I^{n} W\right)$ is stable for $n \in \mathbb{N}^{s}, n \geq k$.

To start, let $P_{1}, \ldots, P_{t}$ denote the primes in $\bigcup_{n \in \mathbb{N} s} A s s\left(\left(U+I^{n} V\right) / I^{n} W\right)$. Take $q \in \mathbb{N}^{s}$ as in Lemma 3.3. If $\left(U+I^{n} V\right) / I^{n} W=0$, for all $n \in \mathbb{N}^{s}, n \geq q$, then clearly $A s s_{R}\left(\left(U+I^{n} V\right) / I^{n} W\right)$ is independent of $n$. If not, there exists $p \geq q$ with $\left(U+I^{p} V\right) / I^{p} W \neq 0$. Increasing $q$ if necessary, we may take $p=q$ and also assume that $\left(U+I^{q} V\right) / I^{q} W \neq 0$. Now take $P \in A s s_{R}\left(\left(U+I^{q} V\right) / I^{q} W\right)$; so $P=P_{i}$, for some $1 \leq i \leq t$. Write $P=\left(I^{q} W: c\right)$, with $c \in U+I^{q} V$. By Lemma 3.3, for any $1 \leq j \leq s$, either $P=\left(I^{q+e^{j}} W: I_{j} c\right)$ or $P=\left(I^{q+e^{j}} W: a\right)=(0: a)$, with 
$a \in U \cap\left(0:_{T} P\right)$. It follows immediately that $P \in A s s_{R}\left(\left(U+I^{q+e^{j}} V\right) / I^{q+e^{j}} W\right)$ for all $j$. Continuing, we get $A s s_{R}\left(\left(U+I^{n^{1}} V\right) / I^{n^{1}} W\right) \subseteq A s s_{R}\left(\left(U+I^{n^{2}} V\right) / I^{n^{2}} W\right)$ whenever $q \leq n^{1} \leq n^{2} \in \mathbb{N}^{s}$. It follows from this and the fact that the union of the primes in $\operatorname{Ass}_{R}\left(\left(U+I^{n} V\right) / I^{n} W\right)$ is finite that there exists $k \in \mathbb{N}^{s}$ such that $A s s_{R}\left(\left(U+I^{n} V\right) / I^{n} W\right)$ is stable for $n \geq k$.

As a corollary we have asymptotic stability of the associated primes of Ext and Tor modules derived from $M / I^{n} M$.

Corollary 3.5. Let $R$ be a Noetherian ring, $I_{1}, \ldots, I_{s}$ ideals of $R, N, M$ finitely generated $R$-modules, and $M^{\prime} \subseteq M$ a submodule. Fix $i \geq 0$. Then there exists $k \in \mathbb{N}^{s}$ such that for all $n \in \mathbb{N}^{s}, n \geq k$, the sets $\operatorname{Ass}_{R}\left(\operatorname{Ext}_{\mathrm{R}}^{\mathrm{i}}\left(N, M / I^{n} M^{\prime}\right)\right)$ and $A s s_{R}\left(\operatorname{Tor}_{\mathrm{i}}^{\mathrm{R}}\left(N, M / I^{n} M^{\prime}\right)\right)$ are stable.

Proof. Since the proofs for Ext and Tor are essentially the same, we prove the corollary for the Ext modules. But this is clear, since if we take a free resolution of $N$ by finitely generated free $R$-modules and "Hom" this resolution into $M / I^{n} M^{\prime}$, at the $i$ th place in the resulting complex, we have a short complex of the form $A / I^{n} A^{\prime} \rightarrow B / I^{n} B^{\prime} \rightarrow C / I^{n} C^{\prime}$. Hence, the previous proposition applies.

Remark 3.6. We cannot expect asymptotic stability of primes associated to the Ext modules if we interchange the arguments $N$ and $M / I^{n} M^{\prime}$. Indeed, [Si] provides an example of a local cohomology module $H_{J}^{i}(N)$ having infinitely many associated primes. Since the associated primes in a direct limit of modules are contained in the union of the associated primes of the modules comprising the limit, it follows that in this case the union over $t \in \mathbb{N}$ of $\operatorname{Ass}_{R}\left(\operatorname{Ext}_{\mathrm{R}}^{\mathrm{i}}\left(R / J^{t}, N\right)\right)$ is not even finite.

\section{A THRESHOLD FOR STABILITY OF ASYMPTOTIC PRIMES}

Throughout this section $R$ will be a Noetherian standard $\mathbb{N}^{d}$-graded ring and $A$ will be a finitely generated graded $R$-module. In [W], the second author showed that there exists $k \in \mathbb{N}^{d}$ such that $\operatorname{Ass}_{R_{0}}\left(A_{m}\right)$ is stable for all $m \in \mathbb{N}^{d}, m \geq k$. In this section we refine the results in $[\mathrm{W}]$ in order to give an explicit $k$. We begin by recalling two lemmas.

Lemma 4.1 ([W, 3.1]). There exists $l \in \mathbb{N}^{d}$ such that for each $1 \leq i \leq d$, we have $\left(0:{ }_{A} R_{e^{i}}\right) \cap A_{m}=0$ whenever $m \in \mathbb{N}^{d}$ satisfies $m_{i} \geq l_{i}$.

Lemma $4.2([\mathrm{~W}, 3.2])$. Suppose $P \in \operatorname{Ass}_{R_{0}}\left(A_{m}\right)$ for some $m \in \mathbb{N}^{d}, P=\left(0: R_{0} x\right)$. Then there exists a homogeneous element $a \in R$ such that $\mathcal{P}:=\left(0:_{R}\right.$ ax $)$ is prime (so $\mathcal{P}$ belongs to $A s s_{R}(A)$ ) and $\mathcal{P} \cap R_{0}=P$.

We omit the proofs, but would like to point out that the statements and proofs in this section tacitly allow for the possibility that for some $m$, and hence all large $m, A_{m}=0$ and $A s s_{R_{0}}\left(A_{m}\right)=\emptyset$.

Lemma 4.3. Take $l \in \mathbb{N}^{d}$ as in Lemma 4.1. Suppose that $m \in \mathbb{N}^{d}$ satisfies $m_{i} \geq l_{i}$ for some $1 \leq i \leq d$. If $P \in A s s_{R_{0}}\left(A_{m}\right)$, then $P=\mathcal{P} \cap R_{0}$ for some $\mathcal{P} \in A s s_{R}(M)$ such that $R_{e^{i}} \nsubseteq \mathbb{P}$. Thus, if $m \geq l$, then every element of $A s s_{R_{0}}\left(A_{m}\right)$ is of the form $\mathcal{P} \cap R_{0}$, for $\mathcal{P} \in A s s_{R}^{+}(A)$.

Proof. Fix $m$ and, without loss of generality, assume $i=1$, so that $m_{1} \geq l_{1}$. Take $P$ in $A_{s s_{R_{0}}}\left(A_{m}\right)$ and write $P=\left(0: R_{0} x\right)$, for $x \in A_{m} \backslash\{0\}$. By Lemma 4.2, we can find homogeneous $a \in R$ such that $\mathcal{P}:=\left(0:_{R} a x\right)$ is a prime ideal and contracts to 
$P$. Since $\operatorname{deg}(a x) \geq m$ and $m_{1} \geq l_{1}$, Lemma 4.1 gives $\left(0:_{A} R_{e^{1}}\right) \cap A_{\operatorname{deg}(a x)}=0$. Thus ax $\notin\left(0::_{A} R_{e^{1}}\right)$. So $R_{e^{1}} \nsubseteq \mathcal{P}$ and the first statement holds. The second statement readily follows.

Lemma 4.4. Suppose $\mathcal{P}=\left(0:_{R} x\right), x \in A_{t}, t \in \mathbb{N}^{d}$. If $R_{e^{i}} \not \subset \mathcal{P}$, then $\mathcal{P} \cap R_{0}$ belongs to $A s s_{R_{0}}\left(A_{t+\alpha e^{i}}\right)$ for all $\alpha \in \mathbb{N}$. Thus, if $\mathcal{P} \in A s s_{R}^{+}(A)$, then $\mathcal{P} \cap R_{0}$ belongs to $A_{s s_{R_{0}}}\left(A_{m}\right)$ for all $m \in \mathbb{N}^{d}$ satisfying $m \geq t$.

Proof. We assume, without loss of generality, that $R_{e^{1}} \nsubseteq \mathcal{P}$. Take $a \in R_{e^{1}}, a$ not in $\mathcal{P}$. Then $\mathcal{P}=\left(0:_{R} x\right)=\left(0:_{R} a^{\alpha} x\right)$ for all $\alpha \in \mathbb{N}$, which immediately gives what we want. The second statement also now follows at once.

We now can give the required $k \in \mathbb{N}^{d}$. Note that the proposition also describes explicitly the stable set of primes.

Proposition 4.5. Let $R$ be a Noetherian standard $\mathbb{N}^{d}$-graded ring and let $A$ be a finitely generated $\mathbb{N}^{d}$-graded $R$-module. Let $A s s_{R}^{+}(A)=\left\{\mathcal{P}_{1}, \ldots, \mathcal{P}_{r}\right\}$ and write $\mathcal{P}_{i}=\left(0:_{R} c_{i}\right)$ with $\operatorname{deg}\left(c_{i}\right)=q^{i} \in \mathbb{N}^{d}, 1 \leq i \leq r$. Take l as in Lemma 4.1. If we set $k:=\sup \left\{q^{1}, \ldots, q^{r}, l\right\}$, then for all $m \geq k$, we have $\operatorname{Ass}_{R_{0}}\left(A_{m}\right)=A s s_{R_{0}}\left(A_{k}\right)=$ $\left\{\mathcal{P}_{1} \cap R_{0}, \ldots, \mathcal{P}_{r} \cap R_{0}\right\}$.

Proof. Since $k \geq q^{i}$ for all $i, \mathcal{P}_{i} \cap R_{0}$ belongs to $\operatorname{Ass}_{R_{0}}\left(A_{m}\right)$ for all $m \geq k$, by Lemma 4.4. On the other hand, since $k \geq l$, Lemma 4.3 shows that for $m \geq k$, every element of $\operatorname{Ass}_{R_{0}}\left(A_{m}\right)$ is of the form $\mathcal{P}_{i} \cap R_{0}$, for some $1 \leq i \leq r$.

Remark 4.6. Of course the $k$ in Proposition 4.5 need not be optimal, but we cannot do any better in this generality. On the one hand, if $F$ is a field and $R=A$ is any standard $\mathbb{N}$-graded $F$-algebra with $R_{n} \neq 0$ for all $n$, then $A s s_{R_{0}}\left(A_{n}\right)$ is the zero ideal of $F$ for all $n$, regardless of the degrees of the annihilators of the elements in $A_{s s}^{+}(A)$. On the other hand, let $p \neq q \in \mathbb{Z}$ be primes and let $R$ be a polynomial ring over $\mathbb{Z}$, with the usual grading and with at least three variables. Take $F, G \in R$ such that $F$ and $G$ are homogeneous, $G$ is irreducible modulo $p$, $F$ is irreducible modulo $q$, and $(F, G) R$ is a prime ideal. For example, one could take $R:=\mathbb{Z}[S, T, U, V, W, X, Y, Z], F:=S T^{d}+U V^{d}$ and $G:=W X^{e}+Y Z^{e}$. Then it is not hard to see that for $A:=R /(p F, q G) R, P:=(p, G) R$ and $Q:=(q, F) R$, $P=\operatorname{ann}(q F), Q=\operatorname{ann}(p G),(F, G)=\operatorname{ann}(p q)$ and $A s s_{R}(A)=A s s_{R}^{+}(A)=$ $\{P, Q,(F, G)\}$. Thus, since $p \mathbb{Z}$ is not associated to $A_{n}$ for $n<\operatorname{deg}(F)$ and $q \mathbb{Z}$ is not associated to $A_{n}$ for $n<\operatorname{deg}(G)$, Proposition 4.5 implies that $A s s_{R_{0}}\left(A_{n}\right)$ does not stabilize until $n$ reaches $k$, which is $\max \{\operatorname{deg}(F), \operatorname{deg}(G)\}$. Note also by Proposition 4.5 that the stable value of $A s s_{R_{0}}\left(A_{n}\right)=\{p \mathbb{Z}, q \mathbb{Z},(0)\}$.

\section{The MAIN RESUlts}

In this section we will prove the results alluded to in the introduction (and stated explicitly in the abstract). We start with a Noetherian standard $\mathbb{N}^{d}$-graded ring $R$ and homogeneous ideals $I_{1}, \ldots, I_{s} \subseteq R$. As in section 3, our main result will be given for the homology modules $H(n)$ derived from complexes of the form $A / I^{n} A^{\prime} \rightarrow B / I^{n} B^{\prime} \rightarrow C / I^{n} C^{\prime}$, where all modules are now $\mathbb{N}^{d}$-graded. As before, we will exploit the fact that for large $n$ the $H(n)$ have the form $\left(U+I^{n-q} V\right) / I^{n-q} W$, which are also $\mathbb{N}^{d}$ - graded. By Proposition 4.5, we therefore need to control the degrees of various elements that are annihilated in these modules as $n \in \mathbb{N}^{d}$ varies. Most of the ingredients for this are in place; we just need to organize the details. 
We begin with a lemma recording a couple of facts about the types of functions we are interested in.

Lemma 5.1. Let $R$ be a Noetherian standard $\mathbb{N}^{d}$-graded ring, $I_{1}, \ldots, I_{s}$ homogeneous ideals and $T$ a finitely generated $\mathbb{N}^{d}$-graded $R$-module.

(1) Let $C \subseteq T$ be a graded submodule and fix $k \in \mathbb{N}^{s}$. Then there is a linear function $h: \mathbb{N}^{s} \rightarrow \mathbb{N}^{d}$ and, for each $n \in \mathbb{N}^{s}$ with $n \geq k$, a set of homogeneous generators for $I^{n-k} C$ of degrees less than or equal to $h(n)$.

(2) Fix $n^{0} \in \mathbb{N}^{s}$ and set $S:=\left\{n \in \mathbb{N}^{s} \mid n \geq n^{0}\right\}$. For $1 \leq j \leq s$ and $0 \leq i<\left(n^{0}\right)_{j}$, set $S_{j, i}:=\left\{n \in \mathbb{N}^{s} \mid n_{j}=i\right\}$. Suppose that $g: S \rightarrow \mathbb{N}^{d}$ and $g_{j, i}: S_{j, i} \rightarrow \mathbb{N}^{d}$ are given by linear functions. Then there exists a linear function $f: \mathbb{N}^{s} \rightarrow \mathbb{N}^{d}$ such that $f(n) \geq g(n)$, if $n \in S$ and $f(n) \geq g_{j, i}(n)$, if $n \in S_{j, i}$.

Proof. For the first statement, fix homogeneous generating sets for $I_{1}, \ldots, I_{s}$ and $C$. Take $a^{1}, \ldots, a^{s}, b$ in $\mathbb{N}^{d}$ such that $a^{j}$ is greater than or equal to the degrees of the generators of $I_{j}$ and $b$ is greater than or equal to the degrees of the generators of $C$. Let $A$ be the $s \times d$ matrix over $\mathbb{N}$ whose rows are the $a^{j}, 1 \leq j \leq s$. Then for all $r \in \mathbb{N}^{s}, I^{r} C$ has a set of generators whose degrees are bounded by $r \cdot A+b$. Thus, for $n \geq k, I^{n-k} C$ is generated in degree $h(n):=(n-k) \cdot A+b$ or less.

For the second statement, let $A, A(j, i)$ be $s \times d$ matrices over $\mathbb{N}$ and $b, b^{j, i} \in \mathbb{N}^{d}$ be such that $g(n)=n \cdot A+b$ for $n \in S$ and $g_{j, i}(n)=n \cdot A(j, i)+b^{j, i}$ for $n \in S_{j, i}$. If we set $C:=A+\sum A(j, i)$ and $d:=b+\sum b^{j, i}$, then $f(n):=n \cdot C+d$ has the required property.

Proposition 5.2. Let $R$ be a Noetherian standard $\mathbb{N}^{d}$-graded ring, $I_{1}, \ldots, I_{s}$ homogeneous ideals, and $T$ a finitely generated $\mathbb{N}^{d}$-graded $R$-module. Let $U, V, W$ be $\mathbb{N}^{d}$-graded submodules of $T$ such that $W \subseteq V$. Then there exists $n^{0} \in \mathbb{N}^{s}$ and a linear function $g: \mathbb{N}^{s} \rightarrow \mathbb{N}^{d}$ such that for all $n \in \mathbb{N}^{s}, n \geq n^{0}$,

$$
A s s_{R_{0}}\left(\left[\left(U+I^{n} V\right) / I^{n} W\right]_{m}\right)
$$

is stable for all $m \in \mathbb{N}^{d}, m \geq g(n)$.

Proof. We adopt the notation of Proposition 4.5 with $A(n):=\left(U+I^{n} V\right) / I^{n} W$. We start by taking $p \in \mathbb{N}^{s}$ such that $A s s_{R}(A(n))$ is stable for $n \in \mathbb{N}^{s}, n \geq p$, which we can do by the proof of Proposition 3.4. In particular, $A s s_{R}^{+}(A(n))$ is independent of $n$, for $n \geq p$. Let $\left\{\mathcal{P}_{1}, \ldots, \mathcal{P}_{r}\right\}$ be this latter set and write $P_{i}:=\mathcal{P}_{i} \cap R_{0}$, for $1 \leq$ $i \leq r$. Then (with $n \geq p$ ), $\left\{P_{1}, \ldots, P_{r}\right\}$ is the stable set of primes $A s s_{R_{0}}\left([A(n)]_{m}\right)$, for $m>>0$, by Proposition 4.5.

Now take $q$ satisfying conditions (1)-(3) in Lemma 3.3. Without loss of generality, we may assume $p=q$. Fix $1 \leq i \leq r$ and write $\mathcal{P}_{i}=\left(I^{p} W: c_{i}\right)$ with $c_{i} \in U+I^{p} V$. Suppose there exists $t^{i} \in \mathbb{N}^{s}$ such that $\mathcal{P}_{i}=\left(I^{p+t^{i}} W: a\right)=(0: a)$, with $a \in U \cap\left(0:_{T} \mathcal{P}_{i}\right)$ homogeneous. Then for $n \in \mathbb{N}^{s}, n \geq p+t^{i}, \mathcal{P}_{i}=\left(I^{n} W: a\right)$ and the degree of $a$ is independent of $n$. If no such $t^{i}$ exists, then it follows from Lemma 3.3 that for all $r^{i} \in \mathbb{N}^{s}, \mathcal{P}_{i}=\left(I^{p+r^{i}} W: I^{r^{i}} c_{i}\right)$. Thus for $n \in \mathbb{N}^{s}, n \geq p$, $\mathcal{P}_{i}=\left(I^{n} W: c_{i}(n)\right)$ where $c_{i}(n)$ is one of the homogeneous generators of $I^{n-p} \cdot c_{i}$ chosen together with a linear function $h_{i}: \mathbb{N}^{s} \rightarrow \mathbb{N}^{d}$ satisfying $\operatorname{deg}\left(c_{i}(n)\right) \leq h_{i}(n)$, which can be done by Lemma 5.1.(1). In either case, we have for each $i, k_{i} \in \mathbb{N}^{s}$, a linear function $h_{i}: \mathbb{N}^{s} \rightarrow \mathbb{N}^{d}$ and elements $c_{i}(n) \in U+I^{n} V$, such that for $n \geq k^{i}$, $\mathcal{P}_{i}=\left(I^{n} W: c_{i}(n)\right)$ and $\operatorname{deg}\left(c_{i}(n)\right) \leq h_{i}(n)$. 
We now find a linear function $l: \mathbb{N}^{s} \rightarrow \mathbb{N}^{d}$ such that for all $i,\left[\left(0: A(n) R_{e^{i}}\right)\right]_{m}=0$, whenever $m \in \mathbb{N}^{d}$ satisfies $m \geq l(n)$ (and $n \in \mathbb{N}^{s}$ is sufficiently large). Set $R_{e}:=$ $R_{e^{1}} \cdots R_{e^{d}}$ and notice that since $\left(0:_{A(n)} R_{e^{i}}\right) \subseteq\left(0:_{A(n)} R_{e}\right) \subseteq\left(0:_{T / I^{n} W} R_{e}\right)$, it will suffice to find $b \in \mathbb{N}^{s}$ and a linear function $l: \mathbb{N}^{s} \rightarrow \mathbb{N}^{d}$ such that for all $n \geq b,\left[\left(I^{n} W:_{T} R_{e}\right)\right]_{m}=\left[I^{n} W\right]_{m}$, for $m \geq l(n)$. Let $N$ denote the elements in $T$ annihilated by a power of $R_{e}$ and apply Lemma 3.1 to $T / N$ to find $b \in \mathbb{N}^{s}$ such that for all $n \geq b,\left(I^{n} W:_{T} R_{e}\right)=I^{n-b}\left(I^{b} W:_{T} R_{e}\right)+N \cap\left(I^{n} W:_{T} R_{e}\right)$. By Lemma $5.1(1)$, there exists a linear function $h: \mathbb{N}^{s} \rightarrow \mathbb{N}^{d}$ such that $I^{n-b}\left(I^{b} W:_{T} R_{e}\right)$ is generated in degrees less than or equal to $h(n)$. Notice that if $x \in I^{n-b}\left(I^{b} W:_{T} R_{e}\right)$ and $\operatorname{deg}(x) \geq h(n)+(1, \ldots, 1)$, then $x \in I^{n} W$. Now take $t \geq(1, \ldots, 1) \in \mathbb{N}^{d}$ such that $[N]_{m}=0$ for $m \geq t$. Then if we set $l(n):=h(n)+t$, it follows that for all $n \geq b,\left[\left(I^{n} W:_{T} R_{e}\right)\right]_{m}=\left[I^{n} W\right]_{m}$ for $m \geq l(n)$. In particular, for any such $n$, $\left[\left(0: A(n) R_{e^{i}}\right)\right]_{m}=0$, for all $i$ and all $m \geq l(n)$.

Finally, let $g: \mathbb{N}^{s} \rightarrow \mathbb{N}^{d}$ be any linear function satisfying

$$
g(n) \geq \sup \left\{h_{1}(n), \ldots, h_{r}(n), l(n)\right\} .
$$

Then for all $n \in \mathbb{N}^{s}, n \geq n^{0}:=\sup _{i}\left\{k^{i}, b\right\}$ it follows from Proposition 4.5 that $A s s_{R_{0}}\left([A(n)]_{m}\right)$ is stable for all $m \in \mathbb{N}^{d}, m \geq g(n)$, which is what we want.

We may now state and prove the main result in this paper.

Theorem 5.3. Let $R$ be a Noetherian standard $\mathbb{N}^{d}$-graded ring, $I_{1}, \ldots, I_{s} \subseteq R$ homogeneous ideals and $A \stackrel{\alpha}{\rightarrow} B \stackrel{\beta}{\rightarrow} C$ a complex of finitely generated graded $R$ modules with degree preserving homomorphisms. Suppose $A^{\prime} \subseteq A, B^{\prime} \subseteq B$ and $C^{\prime} \subseteq C$ are graded submodules satisfying $\alpha\left(A^{\prime}\right) \subseteq B^{\prime}$ and $\beta\left(B^{\prime}\right) \subseteq C^{\prime}$. For $n \in \mathbb{N}^{s}$, let $H(n)$ denote the homology of the induced complex

$$
A / I^{n} A^{\prime} \stackrel{\alpha(n)}{\longrightarrow} B / I^{n} B^{\prime} \stackrel{\beta(n)}{\longrightarrow} C / I^{n} C^{\prime} .
$$

Then there exists a linear function $f: \mathbb{N}^{s} \rightarrow \mathbb{N}^{d}$ such that for all $n \in \mathbb{N}^{s}$, Ass $_{R_{0}}\left([H(n)]_{m}\right)$ is stable for $m \in \mathbb{N}^{d}, m \geq f(n)$.

Proof. As in the proof of the Proposition 3.4, there exists $q \in \mathbb{N}^{s}$ such that for $n \geq q, H(n)$ is isomorphic to a module of the form $\left(U+I^{n-q} V\right) / I^{n-q} W$, which in our present case is also $\mathbb{N}^{d}$-graded. Thus, by the previous proposition, there is $n^{0} \in \mathbb{N}^{s}$ and a linear function $g: \mathbb{N}^{s} \rightarrow \mathbb{N}^{d}$ such that for all $n \in \mathbb{N}^{s}, n \geq n^{0}$, $A s s_{R_{0}}\left([H(n)]_{m}\right)$ is stable for $m \in \mathbb{N}^{d}, m \geq g(n)$.

To finish, we must find a linear function $f(n)$ that works for all $n \in \mathbb{N}^{s}$, not just sufficiently large $n$. We prove this by induction on $s$, assuming that we have $n^{0}$ and $g(n)$ as above. If $s=1$, then since $g(n)$ accounts for all but finitely many values of $n \in \mathbb{N}$, we may certainly find a linear function $f$ such that for all $n \in \mathbb{N}, \operatorname{Ass}_{R_{0}}\left([H(n)]_{m}\right)$ is stable for $m \in \mathbb{N}^{d}, m \geq f(n)$. Now suppose the result holds for $s-1$ ideals and all $\mathbb{N}^{d}$-graded complexes of the required type. For each $1 \leq j \leq s$ and $0 \leq i<\left(n^{0}\right)_{j}$, we apply the induction hypothesis with $A^{\prime}$, $B^{\prime}$ and $C^{\prime}$ replaced by $A^{\prime \prime}:=I_{j}^{i} A^{\prime}, B^{\prime \prime}:=I_{j}^{i} B^{\prime}$ and $C^{\prime \prime}:=I_{j}^{i} C^{\prime}$, taking the ideals $I_{1}, \ldots, I_{j-1}, I_{j+1}, \ldots, I_{s}$. For $t \in \mathbb{N}^{s-1}$, let $H_{j, i}(t)$ denote the resulting homology of the complex $A / J_{j}^{t} A^{\prime \prime} \rightarrow B / J_{j}^{t} B^{\prime \prime} \rightarrow C / J_{j}^{t} C^{\prime \prime}$, where we are writing $J_{j}^{t}$ for the power product $I_{1}^{t_{1}} \cdots I_{j-1}^{t_{j-1}} I_{j+1}^{t_{j+1}} \cdots I_{s}^{t_{s-1}}$. Then there exist linear functions $g_{j, i}$ : $\mathbb{N}^{s-1} \rightarrow \mathbb{N}^{d}$ such that for all $t \in \mathbb{N}^{s-1}, A_{s s_{R_{0}}}\left(\left[H_{j, i}(t)\right]_{m}\right)$ is stable for $m \geq g_{j, i}(t)$. Set $S:=\left\{n \in \mathbb{N}^{s} \mid n \geq n^{0}\right\}$ and for $1 \leq j \leq s$ and $0 \leq i<\left(n^{0}\right)_{j}$, set $S_{j, i}:=$ 
$\left\{n \in \mathbb{N}^{s} \mid n_{j}=i\right\}$. For each $j, i$ let $g_{j, i}^{\prime}: S_{j, i} \rightarrow \mathbb{N}^{d}$ denote the function defined by $g_{j, i}^{\prime}(n)=g_{j, i}\left(n_{1}, \ldots, n_{j-1}, n_{j+1}, \ldots, n_{d}\right)$, for $n \in S_{j, i}$. By Lemma 5.1(2) there exists a linear function $f: \mathbb{N}^{s} \rightarrow \mathbb{N}^{d}$ that dominates $g$ restricted to $S$ and the $g_{j, i}^{\prime}$. It now follows that for all $n \in \mathbb{N}^{s}, A s s_{R_{0}}\left([H(n)]_{m}\right)$ is stable for $m \in \mathbb{N}^{d}, m \geq f(n)$. This completes the proof of the theorem.

Our promised results follow as an immediate corollary.

Corollary 5.4. Let $R$ be a Noetherian standard $\mathbb{N}^{d}$-graded ring, $N, M$ finitely generated, $\mathbb{N}^{d}$-graded $R$-modules and $M^{\prime} \subseteq M$ a graded submodule. Let $I_{1}, \ldots, I_{s}$ be homogeneous ideals of $R$. Then there exist linear functions $f, g: \mathbb{N}^{s} \rightarrow \mathbb{N}^{d}$ such that for all $n \in \mathbb{N}^{s}$,

$$
\operatorname{Ass}_{R_{0}}\left(\left[\operatorname{Ext}^{i}\left(N, M / I^{n} M^{\prime}\right)\right]_{m}\right) \text { and } \operatorname{Ass}_{R_{0}}\left(\left[\operatorname{Tor}_{i}\left(N, M / I^{n} M^{\prime}\right)\right]_{m}\right)
$$

are stable for $m \in \mathbb{N}^{d}, m \geq f(n)$ and $m \geq g(n)$, respectively.

\section{ACKNOWLEDGEMENTS}

Both authors have had enjoyable conversations on primary decomposition and asymptotic primes with Yongwei Yao. In particular, Corollary 3.5, which was proven independently by Dr. Yao using different techniques (see $[\mathrm{Y}]$ ), was a favorite topic of those conversations.

The authors would also like to thank the referee for a careful reading of the manuscript and for offering a number of valuable suggestions.

\section{REFERENCES}

[B] M. Brodmann, Asymptotic stability of Ass $\left(M / I^{n} M\right)$, Proc. Amer. Math. Soc. 74 (1979), 16-18. MR 80c:13012

[CHT] S. Cutkosky, J. Herzog, and N. Trung, Asymptotic behaviour of Castelnuovo-Mumford regularity, Compositio Math. 118 (1999), 243-261. MR 2000f:13037

[KMR] D. Katz, S. McAdam and L. J. Ratliff, Jr., Prime divisors and divisorial ideals, J. Pure Appl. Algebra 59 (1989), 179-186. MR 90g:13030

[Ko] V. Kodiyalam, Asymptotic behaviour of Castelnuovo-Mumford regularity, Proc. Amer. Math. Soc. 128 (2000), 407-411. MR 2000c:13027

[KS] A. K. Kingsbury and R. Y. Sharp, Asymptotic behaviour of certain sets of prime ideals, Proc. Amer. Math. Soc. 124 (1996), 1703-1711. MR 96h:13003

[Mc] S. McAdam, Asymptotic Prime Divisors, Lecture Notes in Math., vol. 1023, SpringerVerlag, New York, 1983. MR 85f:13018

[Sh] R. Y. Sharp, Injective modules and linear growth of primary decompositions, Proc. Amer. Math. Soc. 128 (2000), 717-722. MR 2000e:13004

[Si] A. Singh, p-torsion elements in local cohomology modules, Math. Res. Lett. 7 (2000), 165-176. MR 2001g:13039

[Sw] I. Swanson, Powers of ideals. Primary decomposition, Artin-Rees lemma and regularity, Math. Ann. 307 (1997), 299-313. MR 97j:13005

[T] E. Theodorescu, Derived functors and Hilbert polynomials, Math. Proc. Cambridge Philos. Soc. 132 (2002), 75-88. MR 2002j:13018

[W] E. West, Primes associated to multigraded modules, J. Algebra (to appear).

[Y] Y. Yao, Ph. D. Thesis, University of Kansas (2002).

Department of Mathematics, University of Kansas, Lawrence, Kansas 66045

E-mail address: dlk@math.ukans.edu

Department of Mathematics and Computer Science, Benedictine College, Atchison, KANSAS 66002

E-mail address: ewest@benedictine.edu 